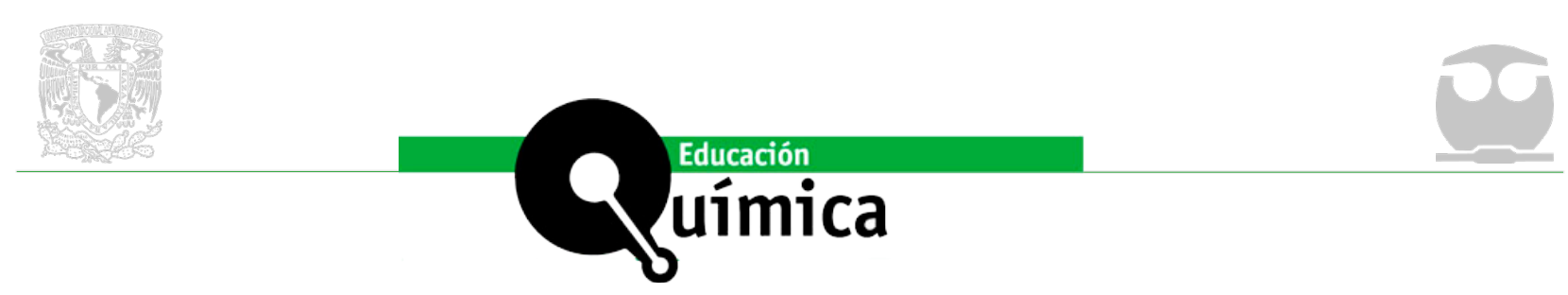

\title{
Aprendiendo reacciones químicas en tiempos de pandemia
}

\author{
Learning chemical reactions in times of pandemic
}

Jorge Pozuelo Muñoz

Recepción 15-07-2021

Aceptación 14-09-2021

\section{Resumen}

Las actividades prácticas son una parte fundamental en la enseñanza de la química. Sin embargo,en un curso como es el último de instituto, no siempre se puede visitar el laboratorio habitualmente. En España, en los cursos 2019-2020 y 2020-21, con la suspensión de las clases presenciales y posterior recomendación de no uso del laboratorio en los institutos, la actividad práctica se ha reducido en su mayor parte. En este artículo se describe una actividad en la que el alumnado preparó y grabó en vídeo actividades experimentales simples sobre reacciones químicas en casa con materiales de uso común, para después visualizarlo y debatirlo entre todos, compartiendo así experiencias prácticas que enriquecieron el contexto formativo de los alumnos en tiempo de pandemia.

\section{Palabras clave}

Reacciones químicas, materiales caseros, enseñanza a distancia, cuarentena

\begin{abstract}
Hands-on activities are a fundamental part of teaching chemistry. However, in a course like last one of high school, it is not always possible to visit the laboratory on a regular basis. In the 2019-2020 and 2020-21 academic years, with the suspension of face-to-face classes and subsequent recommendation not to use the laboratory in the institutes, practical activity has been reduced for the most part. This article describes an activity in which the students prepared and videotaped experiences on chemical reactions at home with commonly used materials, to later visualize and discuss it among all, thus sharing practical experiences that enriched the educational context of the students in pandemic time.
\end{abstract}

\section{Keywords}

Chemical reactions, household materials, remote teaching, quarantine.

${ }^{1}$ Universidad de Zaragoza, España. ORCID: https://orcid.org/0000-0002-9223-6832. 


\section{Introducción}

U no de los conceptos más trabajados de química en la enseñanza secundaria es el de las "reacciones químicas". A su vez, los currículos actuales resaltan la importancia de la experimentación dado que los aspectos positivos de este tipo de actividades, hoy, no son cuestionables (González y Crujeiras, 2016). La actividad experimental promueve el interés por el aprendizaje, ayuda a la comprensión de los conceptos teóricos (Taber, 2015) y facilita la relación entre ellos (Ferrer Roca y Cros Stötter, 2004).

Sin embargo, la situación vivida durante la segunda mitad del curso 2019-2020 debida al confinamiento y la suspensión de las clases presenciales, provocó que las actividades de experimentación en el laboratorio, a priori, se suspendieran (Lupi e Islas, 2021). Ahora bien, el marco educativo que nos ocupa, permite trabajar las reacciones químicasevitando la desconexión existente entre la química que se estudia y la que vivimos en la vida diaria más allá de su carácter anecdótico (Jiménez Liso et al., 2000). Además, la química de lo cotidiano no necesita un laboratorio especial para realizar experiencias prácticas ybasta con materiales sencillos que podemos encontrar en casa (Bueno, 2004). Este tipo de actividades que no necesitan de laboratorio para llevarse a cabo Revertido y Lorenzo (2007) las denominan actividades experimentales simples (en adelante AES).

La actividad que se presenta aquí se ideó con el fin de que el alumnado, en situación de cuarentena, diseñara de forma autónoma un experimento de reacciones químicas con materiales que tuviesen en sus casas, de manera que pusiera a prueba su propio modelode reacción química.

\section{Método}

Esta actividad se llevó a cabo durante la segunda mitad del curso 2019/2020 en el que el que las clases se habían suspendido por la situación sanitaria debida al COVID-19. Los alumnos y alumnas participantes cursan la materia de Química de $2^{\circ}$ de bachillerato de (Anónimo para revisión).

En el contexto de los contenidos que marca el currículo oficial sobre reacciones químicas en $2^{\circ}$ de bachillerato en España(equilibrio químico, reacciones redox y reacciones $\mathrm{pH}$ ), se plantea al alumnado la siguiente actividad: Grabar un vídeo donde se diseñe y explique un experimento químico con materiales de casa. Se limita uno de los reactivos y se les pide que sea el vinagre. Los vídeos de los experimentos serán visualizados por el resto del grupo, y a partir de aquí se pueden generar preguntas sobre las posibles dudas y a su vez el docente puede incidir en los aspectos que considere de mayor relevancia, guiándolos hasta conseguir el aprendizaje buscado.

\section{Resultados}

A continuación, se presentan los experimentos realizados y los resultados.

\section{AES1: Vinagre + tizas + cerilla}

En este experimento una alumna explica cómo influye en una reacción, entre un líquido y un sólido, el grado de división de este último usando vinagre (ácido acético) y tiza común 
FIGURA 1. AES con vinagre + tiza + cerilla.

FIGURA 2. AES pastilla efervescente en agua a mayor y menor temperatura. (principalmente carbonato de calcio). Al experimentar con la tiza más o menos pulverizada, la alumna concluye que aumenta la superficie de contacto entre el sólido y el líquido y la velocidad de reacción se ve afectada. Explicando que la velocidad aumenta cuanto mayor es la superficie de contacto líquido-sólido.

Por otro lado, la alumna platea la manera de comprobar si uno de los productos de reacción en dióxido de carbono, tal como prevé inicialmente. Para ello, introduce una cerilla encendida en el bote, esta se apaga.
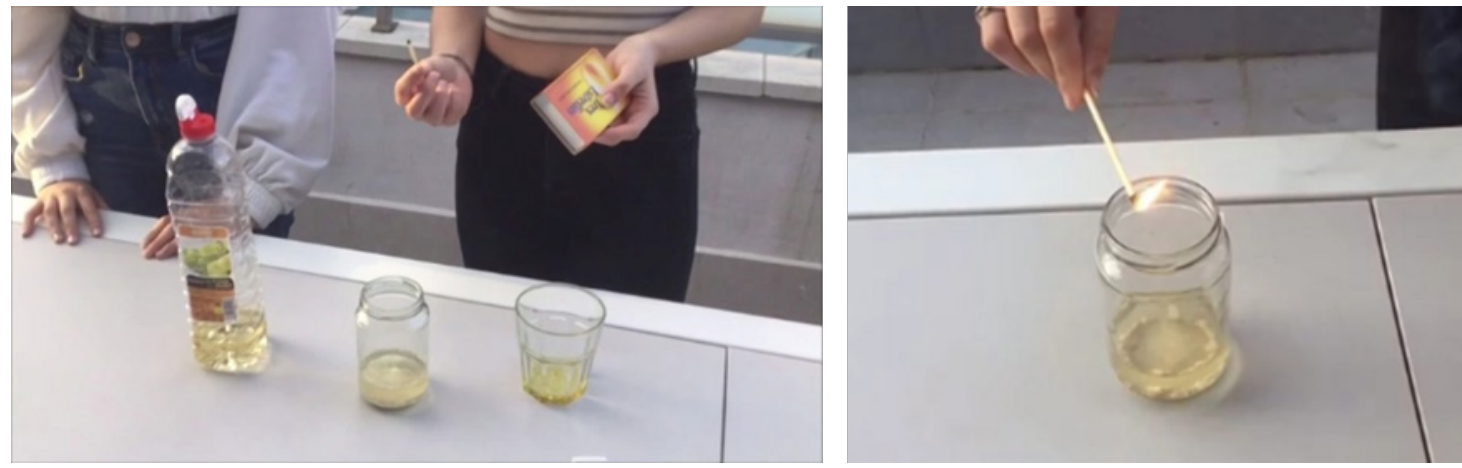

Esto es debido a que, en la reacción entre el vinagre y la tiza, se desprende dióxido de carbono, que es más denso que el oxígeno y desplaza a este fuera del bote. Al introducir la cerilla, esta se apaga por la falta de oxígeno gaseoso, necesario para la combustión. Se puede observar en la figura 1. Las alumnas compartieron la AES a través de la grabación de un video.

Tras la visualización del video por parte de los compañeros, en clase se establecieron relaciones con otros fenómenos de la vida cotidiana, como la disolución más rápida del azúcar en grano que en terrón. A su vez surgieron ejemplos relacionados con la influencia de otras posibles variables, como la temperatura. Se planteó la posibilidad de calentar el vinagre y comprobarlo. Ello dio lugar a probar esta circunstancia, usando la reacción de una "pastilla efervescente" en agua con hielo y agua caliente, observándose que aumentar la temperatura aceleraba la reacción. Se puede observar en la figura 2.
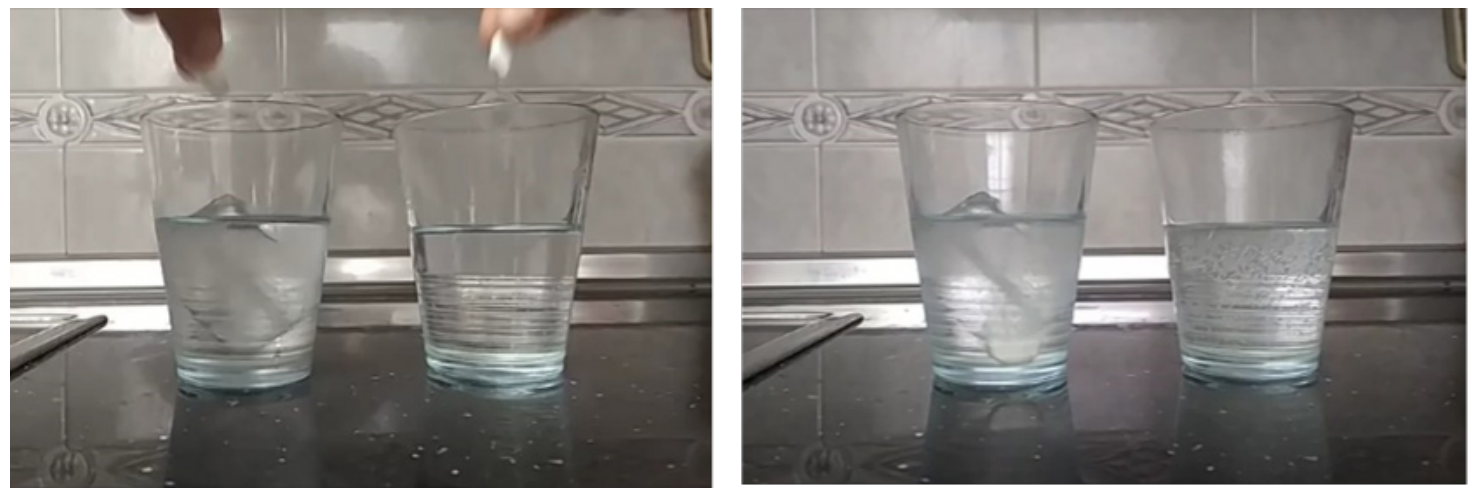

\section{AES2: Vinagre + sal + monedas + clavo}

En esta actividad el alumno cubre monedas de cobre con vinagre, después añade sal para explicar la reacción de oxidación-reducción entre el ácido acético del vinagre y el óxido de 
FIGURA 3. AES de limpiado de monedas con vinagre.

FIGURA 4. AES vinagre con bicarbonato de sodio. cobre de las monedas. Por otro lado, la sal actúa como catalizador, haciendo que el proceso sea más rápido. El alumno explica que una consecuencia observable es que las monedas adquieren el brillo metálico característico, como se puede ver en la Figura 3 y en el video.
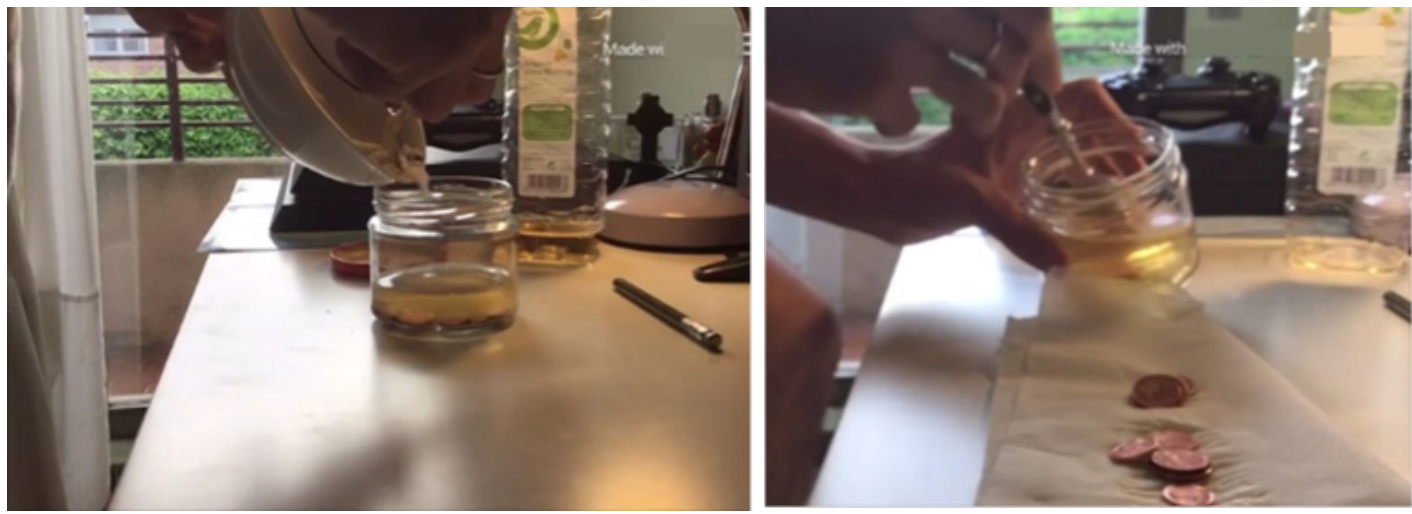

Tras la puesta en común en clase, se pudieron comentar diferentes cuestiones. En primer lugar, se expuso y ajustó la reacción química por el método del ión-electrón. A veces, la teoría relacionada con este tipo de reacciones se convierte en un proceso más que en un concepto, de esta manera, el hecho de realizar una AES sirve para acercar la teoría a la realidad. A su vez se trabajó el concepto de catalizador, dado que resulta complejo de entender al no participar de forma explícita dentro de la reacción y al mismo tiempo ser tan importante en la reacción.

\section{AES 3: Vinagre + bicarbonato + globo}

El siguiente experimento consiste en inflar un globo a partir de una reacción de vinagre con bicarbonato de sodio. A pesar de ser una actividad que puede parecer trivial, no lo resulta pues se pudieron extraer varias conclusiones de interés. En la reacción de bicarbonato de sodio con vinagre se produce una sal (acetato de sodio) y dióxido de carbono. En la figura 4 podemos ver imágenes del experimento.
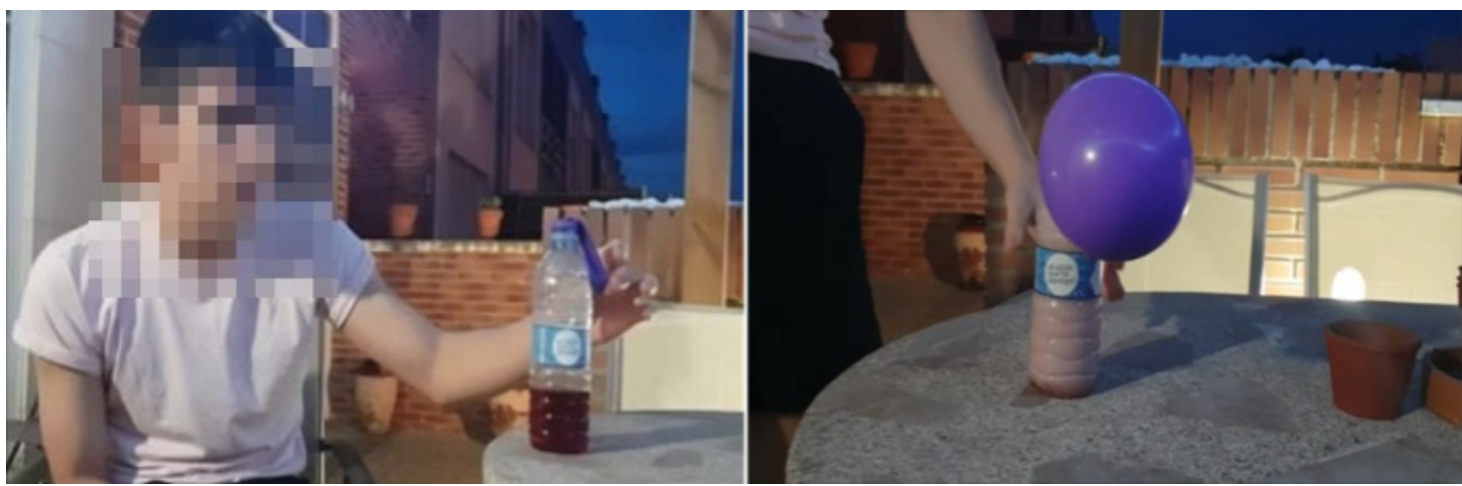

Con este experimento se pudieron trabajar diferentes aspectos. Al finalizar la reacción se observa que el globo se infla incluso más de lo que el propio alumno espera (opta por alejarse del experimento, ver video). Tras comentar en clase este hecho y trabajar con un sistema cerrado (botella+globo), se evidencia que uno de los productos es un gas(dióxido de carbono) y como consecuencia el volumen de los productos es mayor que el de los 
FIGURA 5. AES vinagre con bicarbonato de sodio y una vela. reactivos. Esto sirvió para trabajar la ley de conservación de la materia de Lavoisier, puesto que, aunque la intuición lleva a pensar que se está produciendo más materia, sin embargo, lo único que sucede es que al estar en estado gaseoso parte de ella, ocupa mucho más tal como fueron capaces de concluir los alumnos. El docente sugirió la posibilidad de haber realizado el experimento sobre una balanza, para observar que la masa no cambia. Por otro lado, una vez se produjo la reacción, se observó que aún quedaba líquido en la botella (vinagre), lo cual dio pie a realizar la siguiente pregunta: ¿Si hubiesen añadido más bicarbonato, se habría consumido todo el vinagre? Se introdujo así el concepto de reactivo limitante y la ley de Proust de las proporciones definidas.

\section{AES 4: Vinagre + bicarbonato + vela}

Con esta actividad se trabajan de nuevo la reacción entre bicarbonato de sodio y vinagre en la que se desprende dióxido de carbono gaseoso. Sin embargo, en este caso el sistema es abierto. Ahora bien, debido a que el dióxido de carbono tiene una mayor densidad que el aire, este quedará dentro del recipiente. De esta manera, si volcamos el recipiente levemente sobre una vela encendida, esta se apagará debido al dióxido de carbono (ver Figura 5 y video del desarrollo de la AES).

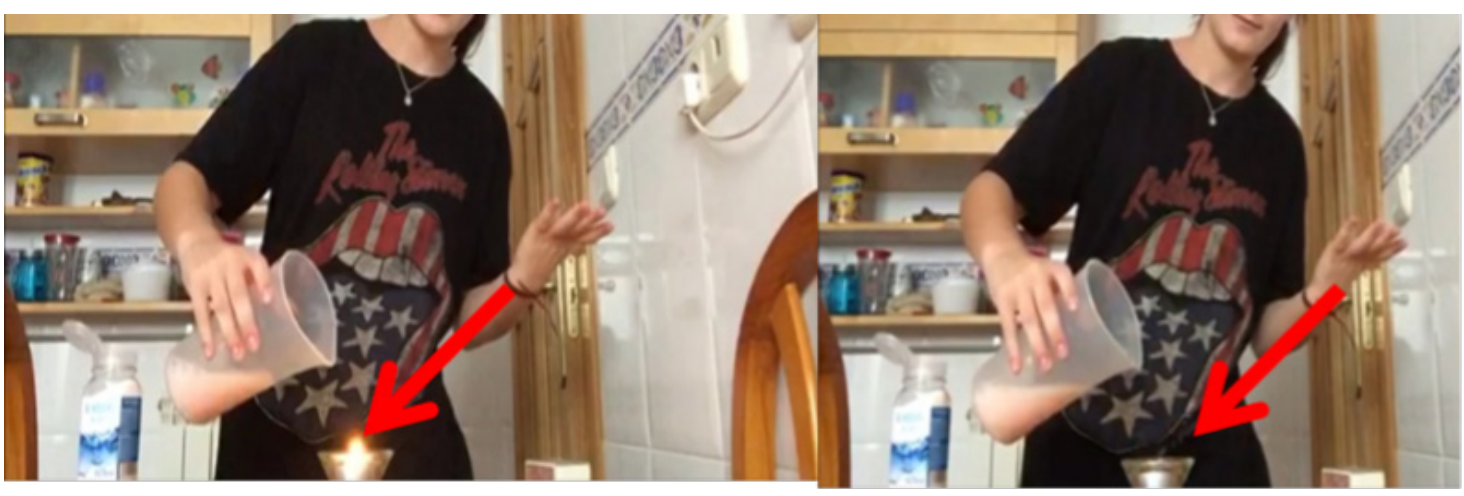

Los temas tratados tras la visualización de este experimento fueron dos principalmente. En primer lugar, mostrar el carácter fluido de los gases. Es común que los alumnos no caractericen los gases como fluidos. Con este experimento se muestra que, debido a la mayor densidad del dióxido de carbono, este cae "al igual que lo haría un líquido invisible" sobre la vela y la apaga, es decir, evidenciamos una característica fluida de un gas. En segundo lugar y fundamental en la AES es: ¿por qué se apaga la vela? En la AES 1, la cerilla se apagaba debido a la ausencia de oxígeno, y esta es la sugerencia que aporta el alumnado en un principio, pero la cuestión ahora es que la vela permanece encendida hasta que se vierte (que no se puede ver) sobre ella el dióxido de carbono. En la combustión de una vela los reactivos son la vela (principalmente parafina, compuesto de carbono e hidrógeno) y oxígeno gaseoso, siendo los productos dióxido de carbono y agua. Al ser una reacción espontánea y contar con productos "de forma ilimitada", no debería dejar de producirse, sin embargo, verter sobre la vela el $\mathrm{CO}_{2}$ provoca el desplazamiento del oxígeno (ausencia de uno de los reactivos), y en consecuencia la reacción se detiene y la vela se apaga. 


\section{Discusión y conclusiones}

A pesar de tener que ejercer una docencia de la ciencia desconocida hasta el momento, pasando de ser presencial a no presencial en cuestión de días, es decir, una docencia adaptada (Grasso, 2021) y forzadamente innovadora (Lupi e Islas, 2021), estas actividades han permitido seguir formando al alumnado en este caso de enseñanzas secundarias. Así, los alumnos han trabajado procedimientos tales comoel diseño de un experimento químico con materiales comunes, lo que evidencia la presencia de la química en su contexto diario (Jiménez Liso et al., 2000). Al mismo tiempo ellos mismos han acercado la teoría a la práctica al tener que usar conceptos teóricos para diseñar sus propios experimentos, lo que ha facilitado su comprensión (Taber, 2015).

En los debates posteriores a la visualización de cada actividad, se ha conseguido una participación activa y la interacción entre ellos (Ferrer Roca y Cros Stötter, 2004), en gran parte motivada por el protagonismo del propio alumnado en la actividad. Esta participación ha llevado a trabajar conceptos diversos en cada AES, fomentando así la conexión entre conceptos teóricos a veces demasiado encapsulados (González y Crujeiras, 2016). Por último, se ha demostrado que a pesar de no poder visitar el laboratorio, en este caso debido a la suspensión de las clases por la COVID-19, se han podido llevar a cabo AES de química que acorten las distancias entre los libros de texto y la realidad(Bueno, 2004). Estas actividades al mismo tiempo han logrado acercar la química al contexto familiar, como se puede observar en algún vídeo donde los familiares hacen de perfectos técnicos de laboratorio y/o cámaras.

\section{Referencias}

Bueno Garesse, E. (2004). Aprendiendo química en casa. Revista Eureka sobre Enseñanza y Divulgación de las Ciencias, 1(1). 45-51. https://revistas.uca.es/index.php/eureka/ article/view/3971/3549

Ferrer Roca, C. y Cros Stötter, A. (2004). La física en el bolsillo: experimentos sencillos de electricidad. Alambique, 39, 79-85.

Grasso, D. H. (2021). Pandemia, docencia y oportunidades. Educación en la Química, 27(01), 95-99. http://educacionenquimica.com.ar/ojs/index.php/edenlaq/article/view/22.

González Rodríguez, L. y Crujeiras Pérez, B. (2016). Aprendizaje de las reacciones químicas a través de actividades de indagación en el laboratorio sobre cuestiones de la vida cotidiana. Enseñanza de las Ciencias, 34(3), 143-160. http://dx.doi.org/10.5565/ rev/ensciencias.2018.

Jiménez Liso, M. R., Sánchez Guadix, M. Á. y Manuel, E. de. (2000). Aprender química de la vida cotidiana más allá de lo anecdótico. Alambique, didáctica de las Ciencias Experimentales, 28, 53-62.

Lupi, L. e Islas, M. S. (2021). La pandemia como motor de la innovación forzada: una experiencia en química inorgánica en condiciones de ASPO. Educación en la Química, 27(1), 101-109. http://educacionenquimica.com.ar/ojs/index.php/edenlaq/article/ view/24. 
Reverdito, A. M. y Lorenzo, M. G. (2007). Actividades experimentales simples. Un punto de partida posible para la enseñanza de la química. $A D E Q R A, 13(2), 108-121$. http://www. adeqra.com.ar/index.php/contenidos/85-vol-13-nd-2-2007

Taber, K.S. (2015). The role of "practical" work in teaching and learning chemistry. School Science Review, 96(356), 75-83 